\title{
STAATSZIELBESTIMMUNGEN ALS MITTEL DER VERFASSUNGSVERGLEICHUNG
}

\author{
Von HenNing von Wedel
}

Die Verfassungsvergleichung hat als Aufgabe die Analyse und Beschreibung fremder Verfassungsordnungen und deren Vergleichung untereinander oder mit der eigenen Verfassung. Es versteht sich von selbst, daß die Kenntnis der Funktionen und Bewährung ähnlicher Verfassungsinstitutionen in anderen Ländern den Blick für die Funktionsweise, die Vorzüge und Mängel der eigenen Institutionen schärft. Nicht anders ist es mit der Betrachtung völlig unähnlicher Lösungen der Staatlichkeit. Die Erkenntnis, daß andere Völker die ihnen gestellten Aufgaben in ganz anderer Weise angegangen sind und der Versuch, diesen Weg gedanklich nachzuziehen, sind jedenfalls geeignet, der Versuchung, die eigene Verfassungsordnung als optimal und vorbildlich anzusehen, einen gewissen Widerstand entgegenzusetzen. Diese Vorzüge von Verfassungsvergleichung würden aber kaum eine dauerhafte Beschäftigung mit ihr oder gar das Unternehmen einer Zeitschrift rechtfertigen, wenn nicht zugleich eine unmittelbare Auswirkung auf die konkrete Arbeit von Verfassungsjuristen möglich wäre.

Wer sich die Aufgabe eines Verfassunggebers ${ }^{1}$ vergegenwärtigt, der die jeweils adäquate verfassungsrechtliche Lösung für bestimmte Organisationsprobleme seines Volkes und Staates finden soll, erkennt sogleich, welchen Nutzen hier die Ergebnisse der Disziplin Verfassungsvergleichung haben könnten. Voraussetzung ist aber, daß für den Vergleich der Eignung bestimmter verfassungsrechtlicher Institute und Lösungen für das konkrete Organisationsproblem ein Maßstab vorhanden ist, nach dem Geeignetes geortet, Ungeeignetes ausgeschieden werden kann. Einen solWie bei jeder Gesetzgebung stellt sich auch bei der Verfassunggebung die Auf-

Wie bei jeder Gesetzgebung stellt sich auch bei der Verfassunggebung die Aufgabe in der Weise, daß zunächst eine Zieldiskussion zu führen ist, die die Absichten und Intentionen hervortreten läßt. Sodann bedarf es der Entscheidung darüber, welche Ziele verfolgt werden sollen. Erst daran schließt sich dann die Suche nach dem für die jeweilige Ziel- bzw. Zweckverfolgung geeigneten Mittel an. Dieser Vorgang ist bei einfachen Gesetzesvorhaben sofort durchschaubar. Es taucht eine bestimmte regelungsbedürftige Situation auf, z. B. das ständige Ansteigen der Verkehrstoten. Es bedarf hier keiner Zieldiskussion, denn es ist unstreitig, daß Ziel die Reduzierung dieser Zahl - im Optimum auf $0-$ ist. Hier beschränkt sich die gesamte Diskussion auf das "Wie“, auf das Mittel der Herabsetzung. Angesichts des hohen Ziels können auch Zielkonflikte kaum auftauchen, da die Beschäftigungssorgen der Automobilbranche oder das Vergnügen am und natürlich die Freiheit zum schnellen Fahren nicht ernsthaft ins Spiel gebracht werden können. Solche Argumente beeinflussen allenfalls bei der Abwägung verschiedener Mittel deren Auswahl, ohne das Ziel in Frage zu stellen.

\footnotetext{
1 Die Probleme der Verfassunggebung werden neuerdings wieder stärker auch wissenschaftlich bearbeitet. Vgl. etwa in dieser Zeitschrift H. Krüger, Die Kunst der Verfassunggebung VRU 1974, S. 233 ff.; v. Wedel, Das Verfahren der demokratischen Verfassunggebung, Berlin 1976 und die dort erwähnte Literatur. Sowie R. Germann, Politische Innovation und Verfassungsreform, Bern und Stuttgart 1975; U. Häflin, Verfassunggebung in: Probleme der Rechtsetzung, Referate zum Schweizer Juristentag 1974, Basel 1974, S. $75 \mathrm{ff}$.
} 
Ganz anderes Gewicht kommt dagegen der Zieldiskussion bei der Verfassunggebung zu. Denn da gilt es, eine Vielzahl von Zielen in ein Verhältnis zu setzen, insbesondere auch gegenläufige, ja sich sogar gegenseitig ausschließende Ziele auszubalancieren ${ }^{2}$. Es bedarf hier nur des Hinweises auf die Forderung nach Effizienz und Durchsetzungsvermögen der Regierung gegenüber der Forderung nach Begrenzung und Kontrolle der Machtausübung, der Forderung nach schneller und wirksamer Gefahrenabwehr gegenüber der Forderung nach rechtlicher Überprüfbarkeit aller Akte der staatlichen Gewalt, der Forderung nach Sicherung eines sozialen Mindeststandards gegenüber der Forderung nach Begrenzung der staatlichen Lasten, um deutlich zu machen, daß der Verfassunggeber eine sehr umfangreiche Zieldiskussion durchzuführen hat, ehe er in die Diskussion der konkreten Organisationsform eintreten $\mathrm{kann}^{3}$. Der folgende Versuch ist der Untersuchung gewidmet, ob die Staatszielbestimmungen als ein Mittel der Beurteilung verfassungsrechtlicher Institute und Lösungen nutzbar gemacht werden können. Er muß beginnen mit der Umgrenzung des Begriffes, die zugleich die Frage der abstrakten Ableitung von Staatszielen aufwirft und sich dann der Geeignetheit konkreter Staatszielbestimmungen für die Beurteilung von Verfassungsnormen zuwenden ${ }^{3 a}$.

\section{Begriff und Umfang der Staatszielbestimmung}

Mit der Erkenntnis, daß der Staat für den Bürger bereits vorhanden ist, und daß ihm bereits eine große Aufgabenfülle zugewiesen ist, die er auch wahrnimmt, schwindet das Interesse an einer generellen Bestimmung des Staatszweckes und wendet sich den Staatsaufgaben als dem wesentlichen Gegenstand der Sinngebung des Staates $\mathbf{z u}^{4}$. Der Staat als vorgefundene Gegebenheit bedarf nicht so sehr der Rechtfertigung in der Theorie durch die Offenlegung seiner Unentbehrlichkeit für die Befriedigung sehr abstrakt formulierter Zwecke, sondern rechtfertigt sich durch die richtige und pünktliche Erfüllung der ihm zugewiesenen Aufgaben ${ }^{5}$. Hat schon diese Überlegung das Interesse für die Beschäftigung mit den Staatszwecken zurückgedrängt, so führt die folgende zu einer Negierung von allgemein formulierbaren Staatszwecken überhaupt.

Der Staat taucht in den verschiedensten Formen und Gestaltungen auf. Gemeinsam ist den vielfältigen Erscheinungen in der Staatengemeinschaft zunächst nur, daß es sich jeweils um Organisationsformen bestimmter Menschen handelt, die insgesamt eine größere Dichtigkeit der Beziehungen untereinander unterhalten, als mit der Gesamtheit der nicht mit ihnen gemeinsam organisierten Gruppen. Sinnfällig wird dies an dem Begriff der Grenze, die trotz heutzutage häufig sehr großer Durchlässigkeit, die Trennungslinie zwischen diesen Beziehungsgefügen der Kommunikation

\footnotetext{
2 Vgl. dazu R. Germann a. a. O., S. $21 \mathrm{ff}$.

3 Die Tatsache, daß diese Zieldiskussion fast unendlich ist, verhindert wohl meist die Totalrevision von Verfassungen. Verfassungen kommen vielleicht nur dann zustande, wenn das Nichtvorhandensein eine Verfassung fordert oder wenn das Ziel, meist Aufbau eigener Staatlichkeit, sich von selbst versteht. Vgl. zur Zieldiskussion in der Schweiz die vorige Fußnote und K. Höflein a. a. O., S. 76. Die kontroversen Standpunkte in der allgemeinen Staatslehre belegen, daß es keine einheitliche Auffassung

3a Zur Problematik der Messung der Effizienz von Normen in Entwicklungsgesellschaften vgl. Brun-Otto

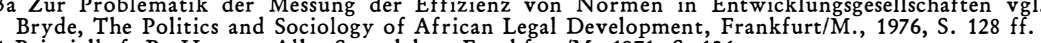

4 Beispielhaft R. Herzog. Allg. Staatslehre, Frankfurt/M. 1971, S. 106.

5 Zur Ermittlung dieser Aufgaben in der Wissenschaft vgl. E. Becker, Wissenschaftliche Erörterungen der Staatsaufgaben in: Menschenwürde und freiheitliche Rechtsordnung, Festschrift für W. Geiger, Tübingen 1974 , S. $755 \mathrm{ff}$. Eine konkrete Untersuchung über "Die Staatsaufgaben nach dem Grundgesetz ${ }^{\alpha}$ hat H. P. Bull vorgelegt. Frankfurt/M. 1975.
} 
und des Austausches bezeichnet. Es handelt sich um Gruppen von Menschen, die zur Daseinsbewältigung zusammengeschlossen und gegeneinander abgegrenzt sind. Damit ist die zentrale Aufgabe anscheinend angesprochen: Daseinsbewältigung. Bei näherer Betrachtung zeigt sich aber, daß diese Aufgabe keine besondere ist, die die staatlich verfaßte Gruppe kennzeichnet, sondern daß es sich um eine Vorgegebenheit des menschlichen Daseins überhaupt handelt. Daseinsbewältigung ist eine Aufgabe für jeden einzelnen, keine Aufgabe, die einer Institution der organisierten Gruppe zugewiesen werden kann.

Aufgabe der staatlich verfaßten Gruppe könnte nun allerdings die Erleichterung und bessere Bewältigung dieser Aufgabe sein, der Staat könnte also instrumental als Mittel der Daseinsbewältigung verstanden werden. Damit wäre aber für die konkrete Frage nach seinen Aufgaben nichts gewonnen, denn der bloß instrumentale Charakter verwiese auf den Benutzer zur Beantwortung der Aufgabenstellung. Die Aufgaben würden wechseln je nach den Anforderungen der Mitglieder, den Bürgern, die gemeinsam das Staatsvolk bilden. Die Anforderungen der Bürger an den Staat, und damit der Einsatz der Institution, sind nicht abstrakt formulierbar. Die konkrete politische Situation im Äußeren wie im Inneren, die Entwicklung der Bedürfnisse und ihrer Befriedigungsmöglichkeiten, sind einem ständigen Wandel unterworfen. Die Betrachtung der ständig sich wandelnden Aufgaben der allgemeinen Verwaltung zeigt, daß eine Festlegung ihrer Zwecke und Aufgaben schnell überholt würde von den Anforderungen der Zeit. $\mathrm{Da}$ es sich in jedem Fall um menschliche Veranstaltungen handelt und die Aufgabe des Menschen nun einmal Daseinsbewältigung heißt, ist die Feststellung, daß auch der Staat in all seinen Erscheinungsformen der Daseinsbewältigung zu dienen hat, letztlich nicht mehr als eine Binsenweisheit ${ }^{6}$.

Entscheidend kommt es nicht auf abstrakte Zwecke oder Ziele an, sondern darauf, welche Aufgaben und Ziele in der konkreten Situation für die Daseinsbewältigung zu erfüllen sind. Die abstrakte Betrachtung von Staatszielen und -zwecken wäre nichts anderes als der Versuch der Herausarbeitung bestimmter Grundbedürfnisse, die immer zu befriedigen wären. Dies ist aber mit Sicherheit nicht die Aufgabe der Rechtswissenschaft. Die abstrakte Beschäftigung mit Staatszielbestimmungen ist damit als Gegenstand rechtswissenschaftlicher Betrachtung von vornherein auszuschließen.

Der Begriff der Staatszielbestimmung ist in die verfassungsrechtliche Literatur erst kürzlich wieder eingeführt worden, jedoch nicht im Sinne einer allgemeinen Bestimmung der Ziele des Staates, wie es die Theorien vom Staatszweck versuchen $^{7}$, sondern eben in dem Sinne der Feststellung der vom Verfassungsgeber getroffenen Zielauswahl und Lösung von Zielkonflikten ${ }^{8}$. Diesen Begriff geringerer Reichweite gilt es nunmehr zu betrachten.

6 Diese Feststellung bedeutet nicht etwa eine Schmälerung der wissenschaftlichen Leistung insbesondere Forsthoffs, mit der Daseinsvorsorge die wahre Aufgabe der Verwaltung in das Blickfeld gerückt zu haben. Dieser Fortschritt ist aber nur im Zusammenhang mit der vorherigen Begrenztheit der Verwaltungszwecke erfaßbar. Vgl. dazu E. R. Huber "Vorsorge für das Dasein “ in Festschrift für Forsthoff, München 1972, S. $139 \mathrm{ff}$., R. Herzog, Allg. Staatslehre, S. 116.

7 Vgl. dazu ausführlich Hans Hug, Die Theorien vom Staatszweck. Diss. Zürich 1954; K. Hespe, Zur Entwicklung der Staatszwecklehre in der deutschen Staatsrechtswissenschaft des 19. Jh. Köln/Berlin 1964.

8 U. Scheuner, "Staatszielbestimmungen“ in Festschrift für E. Forsthoff, München 1972, S. $325 \mathrm{f}$.; Krüger, Der Verfassungsgrundsatz, ebenda, S. $187 \mathrm{ff}$., zweifelt an der Brauchbarkeit der Herausarbeitung von sogenannten Leitgrundsätzen und Zielvorstellungen für die Interpretation von Verfassungen, besonders S. $196 \mathrm{ff}$. 
Der Verfassunggeber kann die Aufgaben des Staates, den er konstituieren will, in unterschiedlicher Weise zu bestimmen suchen. Vor allem kommen zwei Arten in Betracht: a) Die Methode der Generalzuweisung aller Aufgaben und Ausgrenzung von Teilbereichen. Es wird also prinzipiell vermutet, daß eine Aufgabe dem Staate zufällt, sofern nicht eine besondere Vorschrift die Aufgaben an andere Stellen zuweist. Diese Technik ist beliebt bei Kompetenzabgrenzungen zwischen dem Zentralregime und Länderorganen in Bundesstaaten ${ }^{9}$.

Beschränkt man dagegen die Staatsaufgaben auf die dem Staat jeweils konkret zugewiesenen Aufgaben, so muß die Übernahme neuer Aufgaben jeweils mit dem Nachweis der Übertragung dieser Aufgabe durch die Verfassung an den Staat gerechtfertigt werden. Zumindest die deutsche Staatsrechtslehre hat sich wohl unter dem Einfluß des Subsidiaritätsprinzips dieser Auffassung verschrieben ${ }^{10}$. Die Bemühungen um die Ergründung von Verfassungsaufträgen und Verfassungsprinzipien, wie die ganze Lehre von der Aufgabenzuweisung durch Kompetenzzuweisung, beweisen dies ${ }^{11}$. Allerdings geht es dabei keineswegs um die Verwirklichung von Intentionen der Verfassungsgeber ${ }^{12}$, sondern um die Legitimation beliebiger Vorhaben, die politisch leichter durchführbar sind, wenn sie sich mit der Aura des Verfassungsvollzuges umgeben können. Unabhängig von bestimmten Ausuferungen läßt sich jedoch feststellen, daß nach allgemeinem Verständnis in Deutschland jegliches staatliches Handeln zumindest einer implizit gegebenen Ermächtigung bedarf.

Diese Auffassung befriedigt zwar besser als die erste die Ansprüche nach objektiver Rechtsstaatlichkeit, birgt aber die Gefahr, dem Staat gerade dann die „Kompetenz" $\mathrm{zu}$ versagen, wenn völlig unerwartete und unvorstellbare Lagen schnelles Reagieren und die Erfüllung neuer Aufgaben erfordern. Die plötzliche alarmierende Verschlechterung der elementaren Daseinsvoraussetzungen, wie Luft und Wasser in den Industriestaaten, bieten dafür ein instruktives Beispiel, dessen Bedrohlichkeit in Deutschland nur dadurch gemildert wird, daß wenigstens eine Länderkompetenz für diese Aufgabenstellung besteht. Vorzuziehen dürfte sein die generelle Aufgabenzuweisung an den Staat, alles für die Daseinsvorsorge Wichtige zu leisten und nicht so sehr auf objektive Aufgabenbegrenzung, sondern auf die Interorgankontrolle zu vertrauen.

Verfassunggeber verzichten trotzdem selten auf die Festlegung zumindest sehr allgemeiner Staatsziele. Man kann hier in erster Linie generelle Ziele und konkrete Ziele unterscheiden.

Generelle Ziele sind solche, die auf Lagen und Probleme gemünzt sind, die als dauernd vorhanden gedacht werden. Als solche generellen Staatsziele werden meist Sicherheit, Frieden, Rechtsstaatlichkeit, Sozialstaatlichkeit, Sicherung des wirt-

9 Geht man mit H. Krüger, Allg. Staatslehre, 2. April 1966, S. 760, von der prinzipiellen Unbegrenzbarkeit der Staatsaufgaben - allerdings gemildert durch das Prinzip der Nichtdefinition - so könnten etwa die Grundrechte als besondere Aufgabenzuweisungen an den Bürger verstanden werden und so neben der Abgrenzungsfunktion des Abwehrrechts zugleich mit einem positiven staatsfördernden und damit dem Allgemeinwohl verpflichteten Inhalt gefüllt werden.

$10 \mathrm{Vgl}$. dazu vor allem Bull, a. a. O., S. $190 \mathrm{ff}$., der zwar das Subsidiaritätsprinzip ablehnt, durch eine weitere Grundrechtsauslegung aber mit der A. M. zur faktischen Geltung des Prinzips gelangt.

11 Bull a. a. O., S. $132 \mathrm{ff}$; S Scheuner a. a. O., S. $337 \mathrm{ff}$.; vor allem die Sozialstaatlichkeit wird zur Begründung von Verfassungsaufträgen herangezogen; Hesse, Grundzüge des Verfassungsrechts der BRD, 8. Aufl., Karlsruhe 1975, S. 86 f.; Bull, S. 163 ff.; Zur aktuellen Diskussion der Reichweite des Legali8. Aufl., Karlsruhe 1975 , S. $86 \mathrm{f.;} \mathrm{Bull,} \mathrm{S.} 163 \mathrm{ff}$; Zur aktuellen Diskussion der Reichweite des Legaliund Ideologie in: Dimensionen des Rechts, Gedächtnisschrift für René Marcic, Berlin 1974, S. $723 \mathrm{ff}$.

12 Dies wird bewiesen dadurch, daß schon lange niemand mehr aus Art. 15 GG einen Verfassungsauftrag abgeleitet hat, obwohl dies sicher eher den Intentionen des Verfassungsgebers entsprechen würde, als mancher angebliche Verfassungsauftrag in der heutigen Diskussion um Art. 109, Abs. 4 GG. 
schaftlichen Gleichgewichts und Aihnliches angesprochen ${ }^{13}$. Dabei handelt es sich jedoch keineswegs um echte Staatsziele, sondern größtenteils um bloße Oberbegriffe für bestimmte Verfassungsnormen oder notwendige Verfassungsvoraussetzungen ${ }^{\mathbf{1 4}}$. Ein gutes Beispiel bietet die Rechtsstaatlichkeit. Ihre Forderung bewirkt nichts, wenn sie nicht konkret in der Verfassung verwirklicht ist. Schon ihr Inhalt ist völlig unklar, wenn er nicht durch Einzelnormen ausgefüllt wird. Wieso etwa das Rechtsstaatsprinzip das Verbot der Rückwirkung von Gesetzen fordern soll, bleibt unerfindlich. Es läßt sich allenfalls aus dem Grundsatz der Gesetzmäßigkeit der Verwaltung, der Notwendigkeit der Voraussehbarkeit von Umfang und Ausmaß von Rechtsbeeinträchtigungen und ähnlichen Bestimmungen folgern. Als Zielbestimmung ist die Rechtsstaatlichkeit ungeeignet, wenn nicht zumindest angegeben wird, welches Vorbild erreicht werden soll. Ähnlich verhält es sich mit der Sicherheit, die sowohl äußere, wie auch innere Sicherheit umfaßt. Es versteht sich von selbst, daß ein Staat kein verfassungsgemäßes Leben entfalten kann, wenn er ständig von außen bedroht und überfallen wird, wenn ständig Kräfte, die nicht an die Verfassung gebunden sind (Drittstaaten) oder sich nicht an die Rechtsordnung gebunden fühlen (Rebellen, Selbstjustiz, organisiertes Verbrechen) in die Staatsfähigkeit eingreifen. Sicherheit ist also in einem erheblichen Maße nicht Ziel, sondern Voraussetzung für Staatstätigkeit.

Noch ein anderes Argument macht deutlich, daß es sich bei solchen generellen Staatszielbestimmungen nicht um Ziele handeln kann. Der Begriff des Zieles setzt nämlich voraus, daß ein Weg dorthin zurückzulegen ist, daß also das Ziel noch nicht erreicht ist. Nun wird niemand auf die Idee kommen zu behaupten, die Bundesrepublik sei kein sozialer Rechtsstaat in der Sinngebung, die er durch Rechtslehre und Rechtsprechung gefunden hat. Wer von dem Staatsziel Rechtsstaatlichkeit spricht, meint demnach in Wirklichkeit Ausbau, Verbesserung, Vervollkommnung des Rechtsstaates. Für solche Aufgabe bedarf es aber keiner besonderer Zielbestimmung, sondern sie versteht sich von selbst und wird von einem funktionierenden, politischen System von selbst geleistet. Jedenfalls vermag aber die Staatszielbestimmung der Verbesserung und des Ausbaus der staatlichen Institutionen und der Rechtsordnung nichts Besonderes zu leisten gegenüber dem viel stärkeren Motor des ständigen Kampfes der beteiligten Individuen und Gruppen um Festigung und Ausbau ihrer Funktionen, Ämter und Rechtspositionen. Die gemeinhin als generelle Staatsziele und -aufgaben angesprochenen Verfassungsprinzipien erweisen sich somit als Sammelbegriffe für vorhandene Rechtsinstitute oder als Verfassungsvoraussetzungen.

Staatszielbestimmungen können solche Aufgaben nur dann sein, wenn sie im vorhandenen Staat nicht erfüllt werden, wenn sie desiderata sind. Dies ist insbesondere in vielen neuen Staaten der Fall. Ein Staat, dem eine ausgebaute Rechtsordnung fehlt, mag die Rechtsstaatlichkeit als Ziel angeben und in der Verfassung erste Schritte in dieser Richtung unternehmen. Die sogenannten nominalistischen Verfassungen stellen allgemeine und konkrete Staatszielbestimmungen dar. Darauf wird gleich noch zurückzukommen sein. Zunächst ist festzustellen, daß die Identifizie-

$13 \mathrm{Vgl}$. Scheuner, a. a. O., S. 335 f. Die klassischen Ideale der Französischen Revolution dürften auch zu den Staatszielen dieser Art zu zählen sein. Vergleiche zur Ubernahme dieser Ziele in die Verfassungen der frankophonen Staaten Afrikas, H. Krüger, Brüderlichkeit - das dritte fast vergessene Ideal der Demokratie, in: Festgabe für Th. Maunz, München 1971, S. 251 ff. (FN. 6), zusammengestellt von H. Rogge.

$14 \mathrm{Zu}$ diesem Begriff vgl. H. Krüger, Verfassungsvoraussetzungen und Verfassungserwartungen, in: Festschrift für K. Scheuner, Berlin 1973, S. $285 \mathrm{ff}$. 
rung des Begriffes Staatszielbestimmungen mit Verfassungsgrundsätzen zumindest für die hier verfolgten Zwecke einer Beurteilung der Verfassung am Maßstab der durch sie selbst gesetzten Staatsziele unfruchtbar erscheint.

Der Versuch, aus den generellen Lagen, denen sich ein Staat konfrontiert sieht, abgesehen von der Daseinsbewältigung, konkrete formulierbare Staatsziele abzuleiten, ist bislang negativ verlaufen. Für die Verfassungsvergleichung und die Beurteilung von Verfassungen verbleibt als genereller Maßstab nur die Besinnung auf die Zwecke einer Verfassung selbst. In einer Untersuchung über die Brauchbarkeit der amerikanischen Unionsverfassung für die Anforderungen der modernen Zeit, hat Karl Löwenstein gezeigt ${ }^{15}$, daß dies ein ausgesprochen fruchtbarer Maßstab ist. Er geht dabei von den folgenden Grundsätzen als common-sense-Merkmalen einer brauchbaren geschriebenen Verfassung aus:

1. Es muß eine leistungsfähige Regierung geschaffen werden, die ihre Aufgaben sachgemäß und in angemessener Frist verwirklichen kann ${ }^{16}$.

2. Die Regierung muß verantwortlich sein für ihr Tun und mit positiven wie negativen Sanktionen für Erfolg oder Mißerfolg bedroht sein.

3. Es muß ein wirksamer Schutz der Rechte und Freiheiten der Bürger gegen Beeinträchtigungen von seiten der öffentlichen Gewalten, wie auch machtvoller Privatinteressen, bestehen.

Solche allgemeinen Ziele der Verfassungsgesetze bieten durchaus einen Maßstab für die Beurteilung einer konkreten Verfassungsurkunde und deren jeweiliger Umsetzung in die Wirklichkeit. Dieses Thema soll hier nicht weiter verfolgt werden. Es sind dieses Maßstäbe der Vergleichung und Beurteilung von Verfassungen, die sich aus der allgemeinen Verfassungslehre ergeben. Wir wollen uns nunmehr den vom Verfassunggeber selbst gesetzten konkreten Zielen zuwenden.

\section{Konkrete Staatszielbestimmungen durch den Verfassunggeber}

Der Verfassunggeber kann seine Aufgabe von sehr unterschiedlichen Positionen her angehen. Theoretisch lassen sie sich in zwei unterschiedliche Auffassungen des Zweckes und der Leistungsfähigkeit von geschriebenen Verfassungen sondern. Die eine Ansicht geht davon aus, daß die Aufgabe eines Verfassungsgesetzes darin besteht, den politischen Prozeß, der bereits vorhanden ist, möglichst genau in gesetzliche Form zu bringen, und damit auch für die Zukunft in gleicher Weise reproduzierbar zu machen. Die Normativität der Verfassung wird beschränkt auf den Zwang für Reproduktion des bei der Verfassunggebung mit dem Norminhalt identischen politischen Prozesses. Die andere Auffassung verspricht sich von der Verfassung eine Veränderung der politischen Wirklichkeit. Die Verfassung wird mit dem Ausspruch formuliert, den politischen Prozeß nach ihrem Gehalt zu formen. Die Verfassung soll also den politischen Prozeß ändernd beeinflussen.

Es liegt auf der Hand, daß diese unterschiedlichen Auffassungen in der Praxis nicht so sehr aus theoretischen Erwägungen heraus eingenommen werden, sondern daß die konkrete Lage, in der Verfassunggebung vorgenommen wird, wesentlich die

15 Ketzerische Betrachtungen über die amerikanische Verfassung, in: Der Staat als Aufgabe, Gedenkschrift für Max Imboden, Basel/Stuttgart 1972, S. $233 \mathrm{ff}$

16 Hier scheint mir die Ansatzstelle für eine Beurteilung der Leistungsfähigkeit eines politischen Systems anhand seiner konkreten Aufgaben zu liegen. 
Wahl einer bestimmten Position beeinflußt. In einem Land mit einem funktionierenden politischen System, das weitgehend synchron zur Verfassung verläuft, kommt es nicht entscheidend darauf an, wie hoch die Kraft einer Rechtsnorm veranschlagt wird, die Wirklichkeit ändernd $\mathrm{zu}$ beeinflussen. Völlig anders steht es aber damit, wenn ein politisches System sich erst entwickeln soll, wenn die Verfassung es im wahren Sinne des Wortes konstituieren soll. Hinge der Verfassunggeber der ersten Auffassung an, müßte er entweder eine jeweils nur kurzlebige transitorische Verfassung formulieren ${ }^{17}$, oder von vornherein sein Amt zurückgeben. Die Verfassunggebung in einem noch im Entstehen befindlichen Staatswesen wäre weitgehend sinnlos.

Wenn fast alle jungen Staaten sich sogleich eine Verfassung gegeben haben, ja häufig sogar die Verfassunggebung als notwendige Voraussetzung der Staatswerdung angesehen worden ist, so ist von den Verfassunggebern offensichtlich die zweite Position bezogen worden. Die Verfassung wird gegeben in der Überzeugung - oder doch jedenfalls der Hoffnung - daß das politische System sich entsprechend dem in der Verfassung enthaltenen normativen Programm entwickeln werde ${ }^{18}$. In diesem Zusammenhang ist darauf hinzuweisen, daß abgesehen von den Fällen einer nachträglichen Anpassung des Verfassungstextes an geänderte Lagen, jede Verfassungsnorm zunächst eine bloß „nominalistische“ im Sinne der Löwensteinschen Systematik ist ${ }^{19}$. Solange nämlich, bis sie in die Wirklichkeit umgesetzt wird, was selbst in klassischen Verfassungsstaaten sehr lange dauern kann und mitunter nie geschieht ${ }^{20}$. Sofern der Verfassunggeber also eine Verfassung zu schaffen hat, die noch keinem gewachsenen politischen System entspricht, muß er notwendig die Verfassung als Programm auffassen und damit wesentliche Staatszielbestimmungen vornehmen. Abgesehen von solchem generellen Ziel, die Staatlichkeit entsprechend dem in der Verfassung niedergelegten Modell zu entwickeln oder überhaupt erst zu schaffen, setzen Verfassunggeber häufig auch sehr konkrete Nahziele, die meist als unbedingt notwendig für die Entwicklung von Staatlichkeit überhaupt oder doch zur Entwicklung des konkreten Verfassungsmodells angesehen werden.

In einigen modernen Verfassungsurkunden finden sich sogenannte Principles of Policy, die versuchen, die dringendsten Aufgaben der neuen Regierung zu umschreiben. Vor allem in Südasien ist dies, angeregt durch das Beispiel Indiens, üblich ${ }^{21}$. In diesen Fällen ist es naheliegend, die Frage zu stellen, ob die Verfassung und ihre Praktizierung nun auch die Verfolgung dieser Ziele ermöglicht und im

17 Was nach allg. Ansicht zu einer Abwertung des Verfassungsgedankens führen soll. Vgl. statt vieler W. Kägi, Die Verfassung als rechtliche Grundordnung des Staates, Zürich 1945, S. 111. Es ist aber wahrscheinlicher, daß die Prätention, dauerhafte Verfassungen zu machen, die dann sich als kurzlebig erweisen, eher geeignet ist, den Verfassungsgedanken abzuwerten als eine ehrlich als Ubergangsverfassung angelegte Verfassung.

18 Eine solche Forderung läßt sich aus dem Sinn und Zweck von Verfassungen an jede Verfassung stellen. Die Verfassung soll nämlich gerade einsehen, daß das politische System und die Gesellschaft sich in einer bestimmten als positiv oder fortschrittlich gedachten Weise entwickeln, wobei die Entwicklung zi echter Staatlichkeit und Verfolgung des allgemeinen Wohls im Sinne der Entwicklung der Fähigkeiten aller Gemeinschaftsmitglieder bereits Programm genug sein kann. Vgl. dazu vor allem $H$. Krüger, aller Gemeinschaftsmitglieder bereits Programm genug sein kann. Vgl. dazu vor allem H. Krüger,
Die Verfassung als Programm der nationalen Integration, in: Festschrift für Berber, München 1973, S. $247 \mathrm{ff}$. und H. Krüger, Die Verfassung als Programm der nationalen Repräsentation, in: Festschrift für E. R. Huber, Göttingen 1973, S. $95 \mathrm{ff}$.

19 K. Löwenstein, Verfassungslehre, 2. Aufl. Tübingen 1969, S. $151 \mathrm{ff}$.

20 Man denke nur an das Oberste Bundesgericht nach Art. 95 Abs. 1 GG alte Fassung, das bis 1968 nicht eingerichtet wurde. Durch die entsprechende Verfassungsänderung $1968 \mathrm{kam}$ es dann ersatzweise zur Bildung des Gemeinsamen Senats. Der italienische Verfassungsgerichtshof, den die Verfassung von 1947 vorschiebt und der erst 1956 eingerichtet wurde, oder die Vorschrift über die Regionen der gleichen Verfassung, die erst nach fast 20 Jahren, statt wie vorgeschrieben, in einem Jahr in die Tat umgesetzt wurde. Vgl. im übrigen die Beispiele bei Löwenstein, Verfassungslehre, S. $158 \mathrm{ff}$.

21 Vgl. Die Verfassung Indiens, Art. 36-51; vgl. Die Verfassung Pakistans von 1962, Part. II, Chapter 2. Vgl. Die Verfassung Sri Lankas von 1972, Art. 16-18; Vgl. Die Verfassung Bangladeshs von 1972, Art. $8-25$. 
besten Falle zu ihrer Erfüllung geführt hat. Der Verfassunggeber hat gewissermaßen einen Maßstab in seine eigene Schöpfung eingebaut. Erreicht die Verfassung die Verfolgung und endliche Erfüllung dieser Ziele nicht, so müßte jedenfalls der Verfassunggeber seine Aufgabe als gescheitert ansehen.

Die Exemplifizierung der Ziele des Verfassunggebers hat aber noch einen weiteren Vorteil für die Verfassungsvergleichung. Sie erlaubt zumindest in einem gewissen Rahmen die Feststellung, ob die Wahl einer bestimmten Staatsform und die Ausgestaltung der Verfassung in der vorgenommenen Weise geeignet ist, die von ihr selbst formulierten Staatsziele zu erreichen. Es könnte nach den Ergebnissen der Systemtheorie und der Institutionenlehre immerhin vermutet werden, daß z. B. die Durchsetzung der Vergesellschaftung aller Produktionsmittel in einer nach Westminster-Modell gestalteten Demokratie ausgesprochen schwierig sein dürfte. Umgekehrt ist eine Radikaldemokratie aller Voraussicht nach wenig geeignet, bürgerlichen Eigentumsvorstellungen zum Durchbruch zu verhelfen. Interessant ist nun, daß gerade in den erwähnten Ländern Südasiens solche Versuche unternommen worden sind. Die Verfassung Sri Lankas (Ceylon) enthält einen ganzen Katalog von Bürgerrechten und -freiheiten, die dem liberalen Staatsdenken entstammen und überantwortet ihn dann zur Ausführung und Bewahrung an eine Radikaldemokratie. Dagegen sah die inzwischen suspendierte Verfassung Bangladeshs die Durchsetzung sozialistischer Zielsetzungen durch eine Demokratie nach Westminster-Vorbild vor. Mögen diese Beispiele auch nicht sehr aussagekräftig sein, weil diese Verfassungsordnungen möglicherweise gar nicht in der Absicht erlassen wurden, sie auch als wirklich maßgebliches Programm der Entwicklung einzusetzen, so zeigen sie doch, in welche Richtung eine Heranziehung der Staatsziele für die Beurteilung der Verfassungsordnung erfolgen könnte. Das Messen der Verfassung an den von ihr selbst gesetzten Ansprüchen würde es zum Beispiel verbieten, eine Verfassungsordnung schon deswegen als gelungen $\mathrm{zu}$ bezeichnen, weil sie das politische System stabilisiert hat, wenn es ihr nicht gelungen ist, die von ihr selbst als dringlich erkannten Aufgaben einer Lösung zuzuführen.

Eine andere Quelle, aus der sich solche Staatszielbestimmungen im Sinne dringlicher und wichtiger Aufgaben entnehmen lassen, sind die Debatten in den Nationalversammlungen. Die politischen Aufgaben, die zu lösen sind, werden immer wieder herangezogen, um bestimmte Verfassungsinstitute $\mathrm{zu}$ wünschen oder $\mathrm{zu}$ verwerfen. Je mehr Aufgaben zu lösen sind, desto stärker wird die Regierungsgewalt ausfallen. Andererseits kann die Notwendigkeit, allgemeinen Konsens für die Bewältigung dringender kontroverser Aufgaben herbeizuführen, zu einer erhöhten Ausgestaltung der Mitwirkung und Teilhabe aller Kreise des Volkes führen.

Schließlich lassen sich solche Staatsziele aus Parteiprogrammen und den Regierungserklärungen der Parteien und den Äußerungen der Männer der ersten Stunde entnehmen. Die dort gestellten Aufgaben sind es, die primär zur Bewältigung oder Verwerfung der Verfassungsordnung führen. Lassen sich diese Ziele mit der neuen Ordnung nicht verwirklichen, so wird auch die Verfassung schnell ihre normative Kraft verlieren, oder sie sogar nie entfalten. Die Vielzahl der kontroversen Probleme in einem jungen Staat läßt zum Beispiel demokratischen Ordnungen nur dann eine Chance, wenn in Gestalt einer großen Führerpersöhnlichkeit oder einer führenden Partei andere als parlamentarische Formen der Identifikation und Konfliktlösung zur Verfügung stehen. Die Verfassung eines neuen Staates wird nur dann als brauchbar zu bezeichnen sein, wenn sie die konkreten Probleme dieses 
jungen Staates zu lösen vermag. Sie ist nicht an einem abstrakten Ideal zu messen, sondern an den direkt an sie herangetragenen Aufgaben. Die insbesondere aus der allgemeinen Verfassungslehre abgeleiteten abstrakten Anforderungen an eine Verfassung, wozu in erster Linie auch dauerhafte Leistung der Integration gehört ${ }^{22}$, braucht die Verfassung eines jungen Staates nicht unbedingt zu erfüllen. Möglicherweise kann die Lösung bestimmter Aufgaben, wie ausreichende Ernährung aller Bürger, Eindämmung der Überbevölkerung, Bekämpfung epidemischer Krankheiten u. ä., in einer bei idealtypischer Betrachtung unzureichenden Verfassungsordnung das Fundament für eine Verfassungsordnung sein, die allen Anforderungen genügt. Dagegen dürfte eine Verfassungsordnung, die idealtypisch hervorragend ist, jedoch die konkreten Aufgaben ihrer Mitglieder nicht löst, wenig Wert haben. Entscheidend kommt es also nicht darauf an, welche Aufgaben eine Verfassung schlechthin zu lösen hat, sondern von welchen Aufgaben man sich konkret durch sie eine Lösung erhofft. Die vom Verfassunggeber selbst oder von den Initiatoren und repräsentativen Vertretern einer Verfassungsbewegung formulierten Staatszielbestimmungen (im Sinne einer Festlegung der dringlichsten Aufgaben, die durch die neue Gesamtordnung oder Einzelnormen zu lösen sind) vermögen sich einen besseren Maßstab für die Beurteilung von Verfassungen und Verfassungsnormen abzugeben als idealtypische Vergleiche. Konkret gewendet bedeutet dies, daß es nutzlos ist, die mangelnde Mitwirkung des Volkes zu beklagen, wenn das Volk in erster Linie von seinem politischen System materielle Daseinsvorsorge erwartet und diese auch geleistet wird. Sind die Bürger erst der ersten Not enthoben, werden sie auch neue und andere Ansprüche an ihre Verfassungsordnung stellen. Eine solche, auf konkrete Aufgaben bezogene Bewertung von Verfassungen, ist jedenfalls geeignet, den Fehler zu vermeiden, in politischer Stabilität, in formaler Legitimation oder in einer perfekten Gewaltenteilung, "Werte an sich" zu sehen. Alle diese Kategorien sind nur deshalb zu „Werten“ geworden, weil sie sich für die Bewältigung sehr konkreter Aufgaben in unserer Verfassungs-Geschichte oder anderswo bewährt haben ${ }^{23}$.

Die Arbeit von H. P. Bull, „Die Staatsaufgaben nach dem Grundgesetz"4 hat gezeigt, daß sich die Staatstätigkeit in einem durchnormierten Staat sehr weitgehend auf die Verfassung zurückführen läßt. Die Methode oder Staatsaufgabenlehre, die er entwickelt, ist auch geeignet, gewisse Akzente anders zu setzen, auf vergessene Aufgaben aufmerksam zu machen, und eine ständige Besinnung auf die Legitimität staatlichen Handelns herbeizuführen. Zweifelhaft bleibt aber, ob nicht eine so aufgesaßte Aufgabenlehre gerade die Erkenntnis der dringenden und vornehmlich zu erfüllenden Aufgaben verdeckt. Für die tatsächliche Lösung von Zielkonflikten gibt diese Methode wenig her. Ziel einer Staatsaufgabenlehre sollte es nicht so sehr sein, alle denkbaren Aufgaben auf ihre Übertragung in der Verfassung an bestimmte Aufgabenträger zu untersuchen, oder umgekehrt alle Verfassungsnormen auf ihre "Aufgabenträchtigkeit" zu untersuchen, sondern vielmehr die Realisierbarkeit der nach allgemeiner Meinung wichtigen und in erster Linie zu erfüllenden Aufgaben in der bestehenden Verfassungsordnung aufzuzeigen, oder auf die Mängel der Verfassungsordnung angesichts dieser Aufgaben hinzuweisen.

22 Besonders hervorgehoben von M. Häflein a. a. O., S. 111.

$23 \mathrm{Vgl}$. z. B. für den Ansatz einer Staatslehre, die unmittelbar aus konkreten Aufgabenstellungen abgeleitet wird, M. Kriele, Einführung in die Staatslehre, Reinbek 1975.

24 Vgl. oben Anmerkung 6. 
Damit sei dieser Versuch abgeschlossen. Als Ergebnis läßt sich feststellen, daß die in der Verfassung selbst oder in den Gründungsdokumenten enthaltenen konkreten Aufgabenstellungen für die Beurteilung der Brauchbarkeit von Verfassungsnormen durchaus geeignet erscheinen. Die Betrachtung hat aber ergeben, daß abstrakte Staatszielbestimmungen oder Verfassungsgrundsätze kaum für die Verfassungsvergleichung fruchtbar gemacht werden können. 